\title{
Infrared Capture of Palm-Vein Blood Vessel Patterns for Human Authentication
}

\author{
Omoyele Akinsowon, Boniface Alese, Olumide Adewale \\ Federal University of Technology, Akure
}

\begin{abstract}
Biometric Technology is fast taking over from the conventional use of ID Cards, PINs, Tokens and Passwords. This is becoming essential because of the issues of forgotten passwords, stolen ID Cards, compromised tokens and spoofed PINs. Palm-print authentication for access control has been deployed in so many areas of security concern in developing countries. Even though it is still in the theoretical stage in Nigeria, this paper seeks to evaluate the stages involved in the processing of palm-print images for identification or human authentication. It focuses on the feature extraction stage which is where the required features are separated from the others. $T$
\end{abstract}

\section{Introduction}

Biometrics is the science of establishing the identity of individuals based on their unique physical or behavioral attributes. Compared to traditional authentication methods such as ID cards (tokenbased) and passwords (knowledge-based), biometric recognition is considered to be more convenient and secure since an individual's biological signatures cannot be easily lost, forgotten or stolen. [1].

In recent times, security issues have become great priority to individuals, communities and countries as a whole. Hence, reliable security measures have become very necessary. In line with this development, biometrics systems have become relevant. Biometric systems are essentially for pattern recognition based on physiological or behavioral characteristics and are applicable in several areas such as e-commerce for example online banking), access control to buildings or certain areas of an environment [2]. Biometric characteristics include fingerprint, iris, retina, gait, voice, palm vein, face and hand geometry.

Before a trait can be classified as biometric, it must have the following features or characteristics:

(i) Universality: This means that every relevant person should have the characteristic, that is every individual should possess the trait

(ii) Uniqueness: This implies that no two persons should have the same characteristics, that is each individual has an original trait/characteristic

(iii) Permanence: It should not change all through the person's lifetime, i.e. the characteristic should be time-invariant.

(iv) Collectability: The characteristic can be measured, that is the trait can be measured quantitatively. This means that the trait can be easily presented to a sensor, located by it, acquired and converted into a quantifiable digital format

(v) Performance: The measurement and verification of the characteristic should be easy and not errorprone; high accuracy and robustness should be achievable; and the measurement should be precise enough to detect the smallest differences in the characteristic between individuals.

(vi) Acceptability: The relevant persons should be willing to accept the biometric system

(vii) Circumvention: The system should not be easy to fool by fraudulent users.

(viii) Distinctiveness: The trait for two different persons should be sufficiently different to distinguish between them. The higher the degree of distinctiveness, the more individual the identifier is.

\section{Palm Vein Biometrics}

Palm vein biometrics, also called vascular biometrics makes use of captured images of the palm vein pattern beneath the skin. The use of vascular pattern was considered as a potential biometric technology in the early 1990s. Shimizu in his paper expounded this potential and then Cross and Smith introduced thermo graphic imaging technology for acquiring the subcutaneous vascular network on the back of the hand for biometric applications later [3]. From that time on, several research efforts have helped to contribute to this biometric technology. In 1997 however, the first practical application of vascular pattern technology was developed by Alex Hwansoo Choi who co-founded BK systems and several improved versions were developed afterwards. [3].

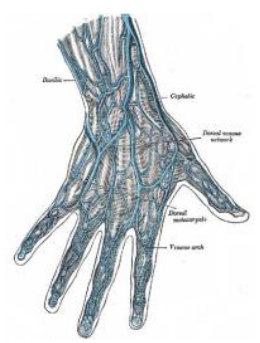

Figure 1. Drawing of the vascular network in the hand. [4] 
In order to obtain clear vein images, only specific blood flow patterns (vessels carrying oxygen-free blood to the heart) are considered. This is done with the aid of infra-red technology for uniquely identifying people [5]. Vascular biometrics relies on capturing an image of the vein pattern beneath the skin with near infrared technology and using that as the basis for individual identification. Unlike fingerprint technology, integrity of the skin is not an issue with vascular biometrics. This technology bypasses the need for quality fingerprints and it's also more conducive in its use when compared with fingerprints biometrics.

Also, the broadness of the palm with its vascular patterns give it a higher advantage over fingerprints for identification. In addition to the earlier mentioned traits are the following:

- Universality

- Uniqueness

- Develops from birth and doesn't change all through the person's life-time

- They carry deoxidized blood towards the heart, except umbilical and pulmonary veins

- The vascular veins are protected internally by the skin

- $\quad$ They are less likely to get damaged

Palm-vein has a vast number of advantages that give it great edge above other biometric traits such as the finger or the back of the hand. For instance, it doesn't have a problem with integrity which is because it is beneath the skin and research has shown that the vein patterns under the palms are unique for every individual, hence it cannot be compromised. Another advantage is that it is non-intrusive [6]. Compared with a finger or the back of a hand, a palm is broader and has more complicated vascular patterns, thus contains more differentiating features for personal identification. The palm is an ideal part of the body for this technology due to absence of hair which can be an obstacle for photographing the blood vessel pattern, and it is less susceptible to a change in skin color unlike a finger or the back of a hand [6].

In this research work, focus is placed on contactless acquisition of palm vein, that is blood vessel patterns. This is made possible as the vein information is hard to duplicate since they are internal to the body. This technology combines the abilities of a device that can read the pattern of the blood veins in the palm without making physical contact and software that can authenticate an individual's identity based on these patterns. Infrared light is used to capture an image of the palm as the hand is held over the sensor device (ITU-T Technology Watch Report, 2009).
In response to the increasing importance of identity verification in many areas of modern life, palm-vein technology has been very useful. For example in places such as electronic government, medical administration systems, and access control systems for secure areas, passenger ticketing, home office and home study environment [7].

\subsection{Palm-Vein Applications}

Palm-vein biometrics is already being implemented in a variety of areas.

2.1.1. Physical access control and time attendance. Palm-vein technology is used for controlling access to rooms or buildings that are for restricted personnel. It is most-widely used for physical access control and attendance. There is processing unit in the control section that authenticates and stores the vein features of enrolled palm-vein. This technology aids employee attendance management and overtime work at large organizations in an effective and efficient manner.

2.1.2. Hospitals. A hospital requires tight security in many areas including medicine cabinets and storage rooms, operating rooms and data centres where patient records are managed and stored .Some sensitive data such as those related to research studies on dangerous virus may be used with dire consequences if it falls into terrorists' hand. Consequently, biometric security methods should be used to protect such sensitive data.

2.1.3. Finance and Banking/Solutions. With the rapid growth of ATM services and credit cards, fraudulent withdrawal of money by using fake or stolen bankcards has become a serious problem. Hand vascular pattern technology can be integrated into banking solutions by two different methods. To address this, palm vein authentication has been utilized for customer confirmation of transactions at bank windows or ATMs. The smart card from the customer's bank account contains the customer's palm vein pattern and the matching software of the palm vein patterns. A palm vein authentication device at the ATM scans the customer's palm vein pattern and transfers it into the smart card. The scanned pattern is then matched with the registered vein pattern in the smart card. Since the preregistered palm vein pattern is not released from the smart card, the security of the customer's vein pattern is preserved. This can be achieved by applying any of these two different methods. In the first method, vascular pattern of customers are stored in the bank's database server. The authentication is carried out by comparing a customer's hand vascular pattern with their enrolled pattern in the database server. In the second method, hand vascular patterns 
of customers are stored in biometric ID cards which are kept by customers. During authentication the customer's hand vascular pattern is compared with the pattern stored in the card for verification. Based on various requirements such as timely response or level of security, banks will decide the appropriate method for their solutions.

2.1.4 Schools. The commonly used ID cards do not offer high level of security because people tend to lose them or fail to return their cards. As a result many universities have adopted hand vascular equipment in research laboratories and private belongings in dormitories. It is not only more costeffective in the long term but also provides an enhanced level of security through individual identification and managerial convenience.

2.1.5. Login authentication. Palm-vein technology can also be integrated into laptop Personal Computers (PC) by Universal Serial Bus (USB). In the event that it is to be used for PC authentication, the existing operating systems and applications can be retained. Also, the sensor can be built into an existing application for enhanced operations [10].

\section{Palm-Vein Technology}

The stages of palm-print authentication include enrollment (which is the actual acquisition of the palm vein), enhancement, extraction, matching and authentication.

An individual's vein pattern image is captured by radiating his/her hand with near-infrared rays. The reflection method illuminates the palm using an infrared ray and captures the light given off by the region after diffusion through the palm.

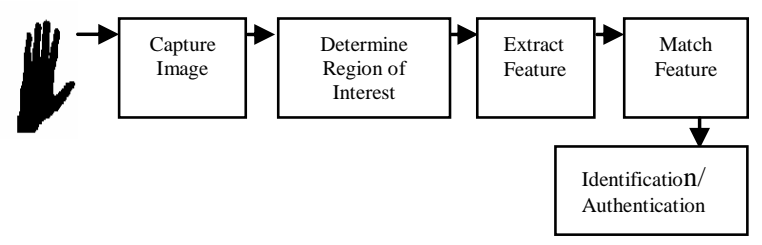

Figure 2. Architecture of a typical pattern recognition system

\subsection{Palm-Vein Acquisition}

An individual's vein pattern image is captured by radiating his/her hand with near-infrared rays. The reflection method illuminates the palm using an infrared ray and captures the light given off by the region after diffusion through the palm.

The relevance of Palm-print for identification, verification or authentication requires a number of stages which also use a wide range of techniques for their actualization. The process starts with acquisition of palm-prints from the relevant people or personnel. In order to obtain clear vein images, only specific blood flow patterns (vessels carrying oxygen-free blood to the heart) are considered. There are three imaging techniques that can be used for capturing vascular images, they are: X-rays, Ultrasonic Imaging and Infrared Imaging. However, Infrared Imaging is preferred over these others because of its non-invasive contactless technique. Also, there are two types of Infrared technologies that can be adopted: the Far-infrared (Far-IR) Imaging and Near-infrared (Near-IR) Imaging technologies. The Far-IR imaging technique captures the thermal patterns emitted by the human body which are unique even to identical twins. Near-IR cameras on the other hand capture radiation in the range $800-1100 \mathrm{~nm}$ of the electromagnetic spectrum. In [8] experiments conducted by Lingu and Leedam show that Near-IR imaging is comparatively less expensive and also gives good quality vein images. They have also found that NearIR imaging is more tolerant to environmental and body conditions and provides a stable image.

Another reason Near-IR is used instead of Far-IR is the problem of image contrast. From experiments carried out by [9], temperature of the surrounding area and the veins are similar leading to similarity in image contrast which makes it difficult to extract vein patterns for comparison. As a result of this problem, the images can only display major veins that have limited details which are inadequate for high security applications. In contrast to this, with Near-IR the captured image is able to display the smaller veins properly. The deoxidized haemoglobin in the vein vessels absorb the infrared rays reducing the reflection rate and causing the veins to appear as a black pattern. The liveliness in the user is detected because the moment the deoxidized haemoglobin ceases to flow through the vein, the pattern will not appear. It is also worthy to note that the ray used in this process is just like sun rays which are not harmful.

The deoxidized hemoglobin in the vein vessels absorbs the infrared ray, thereby reducing the reflection rate and causing the veins to appear as a black pattern. Afterwards, the acquired images are binarized, i.e they are converted to the form that the device can recognize and further processes can be performed on them.

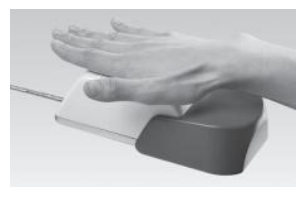

Figure 3. Contactless Acquisition of Palm-Vein 


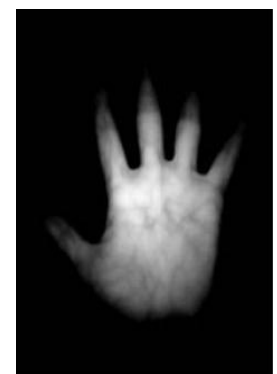

Figure 4 Infrared ray image of palm (www.tiburon-ent.com, 2013)

\subsection{Region of Interest}

In pattern recognition, image pre-processing is usually the first and essential step. In the same light, the first step of image pre-processing is the extraction of the region of interest (ROI). This is the area where the features of the palm that will be used for identification will be selected from. This comes after the image of the palm-vein has been captured. An area of $256 \times 256$ pixels of the enrolled palm vein image is marked out as the ROI. This is the focus area where the features of the palm that will be used for authentication are extracted. As much as the ROI required, the process of selecting the relevant features within this region is of high importance. Hence, to determine the regions of contrast in the ROI, it is high-pass filtered before binarization takes place. The binary image is also median-filtered to remove irrelevant pixels so that the required vein patterns are retained in the ROI.

\subsection{Feature Extraction}

The feature extraction stage is a step to extract the meaningful features from the ROI. At this stage, palm-vein patterns in ROI are studied so that the useful and needed information are derived. The information gathered from this stage is what is used for image processing. [11]. It is worthy to note that the grey level of veins in thermal images is lighter than that of the surrounding skin, under normal conditions [4]. The region of interest is marked out because of is the presence of noise and unwanted features. Hence, the needed important features are filtered for the purpose of achieving the correct identification and authentication. In order to detect the correct edges of the required images, different methods can be employed. However, the most frequently used ones can be classified into two categories: Gradient and Laplacian. The gradient methods detect edges by looking for the maximum and minimum in the first derivative of the image. Examples of gradient method include the Sobel, Prewitt and Roberts Cross operators. The Laplacian method on the other hand searches for zero crossings

in the second derivative of the image. An example is the Marrs-Hildreth operator.

3.3.1. Sobel Egde Detection Operator. This method of locating an edge is characteristic of the "gradient filter" family of edge detection [12]. A pixel location is declared an edge location if the value of the gradient exceeds some threshold. As mentioned before, edges will have higher pixel intensity values than those surrounding them [13]. So once a threshold is set, the gradient value is compared with the threshold value and edges are detected when the threshold is exceeded. The Sobel operator performs a 2-D spatial gradient measurement on an image and so emphasizes regions of high spatial frequency that correspond to edges [12].

Typically it is used to find the approximate absolute gradient magnitude at each point in an input grayscale image. The Sobel edge detector uses a pair of $3 \times 3$ convolution masks, one estimating the gradient in the $\mathrm{x}$-direction (columns) and the other estimating the gradient in the y-direction (rows) [15].

A convolution mask is usually much smaller than the actual image [15]. As a result, the mask is slid over the image, manipulating a square of pixels at a time. Sobel operators are performed to select the maximal value as the resultant value of ROI (region of interest). This operation is operated according to the following expression

\begin{tabular}{|l|l|l|}
\hline+2 & +1 & 0 \\
\hline+1 & 0 & -1 \\
\hline 0 & 1 & -2 \\
\hline
\end{tabular}

$\mathrm{S}_{0}$

\begin{tabular}{|l|l|l|}
\hline+1 & +2 & +1 \\
\hline 0 & 0 & 0 \\
\hline-1 & -2 & -1 \\
\hline \multicolumn{4}{|c|}{$S_{45}$}
\end{tabular}

Figure 5. Sobel Convolution masks

These kernels are designed to respond maximally to edges running vertically and horizontally relative to the pixel grid, one kernel for each of the two perpendicular orientations. The kernels can be applied individually to the input image for separate measurements of the gradient component to be produced in each orientation. These can then be combined together to find the absolute magnitude of the gradient at each point and the orientation of that gradient. They are designed to respond maximally to edges running vertically and horizontally relative to the pixel grid, one for each of the two perpendicular 
orientations [16]. One mask is just the other mask rotated by $90^{\circ}$. The gradient magnitude is given by: $|\mathrm{S}|=\left(\mathrm{S}_{0}^{2}+\mathrm{S} 90^{2}\right)^{1 / 2}$

Typically, an approximate magnitude is computed using:

$|S|=\left|S_{0}\right|+\left|S_{90}\right|$

The direction of the gradient is given by:

$\Theta=\arctan \left(\mathrm{S}_{0} / \mathrm{S}_{90}\right)$

The edge of the pixel, $\mathrm{G}(\mathrm{x}, \mathrm{y})$ is given by:

$\mathrm{G}(\mathrm{x}, \mathrm{y})=|\mathrm{S}(\mathrm{x}, \mathrm{y}) * \mathrm{~S} \mathrm{x}|+|\mathrm{S}(\mathrm{x}, \mathrm{y}) * \mathrm{Sy}|$

Where $S(x, y)$ are the pixels form input image $S$.

3.3.2. Prewitt Edge Detection Operator. The Prewitt filter is very similar to Sobel filter. The $3 \times 3$ total convolution mask is used to detect gradient in the $\mathrm{X}, \mathrm{Y}$ directions as shown in Figure 2. Prewitt filter is a fast method for edge detection. The difference with respect to Sobel filter is the spectral response. It is only suitable for well-contrasted noiseless images [17] .

The Prewitt Edge filter is used to detect edges by applying horizontal and vertical filters in sequence [18]. Both filters are applied to the image and summed to form the final result. The two filters are basic convolution filters of the form:

Horizontal Filter
\begin{tabular}{|l|l|l|l|l|l|}
\hline & 0 & +1 \\
-1 & & \\
\hline-1 & 0 & +1 \\
\hline-1 & 0 & +1 \\
\hline
\end{tabular}

Figure 6. Prewitt Masks

For example, if a $3 \times 3$ window is used

\begin{tabular}{|l|l|l|}
\hline b1 & b2 & b3 \\
\hline b4 & b5 & b6 \\
\hline b7 & b8 & b9 \\
\hline
\end{tabular}

where the centre of the filter is pixel b5, pixel b4 is $(\mathrm{x}-1) \mathrm{y}$, pixel b6 is $(\mathrm{x}-1) \mathrm{y}$; then new $\mathrm{b} 5$ is given by: (b1+b2+b3-b7-b8-b9)+(b3+b6+b9-b1-b4-b7) [19]

3.3.3. Canny Edge Detection Operator. The Canny edge detection algorithm is known to many as the optimal edge detector. Canny's intentions were to enhance the many edge detectors already out at the time he started his work. The Canny edge detector first of all smoothes the image in order to eliminate noise. Afterwards, it finds the image gradient to highlight regions with high spatial derivatives.
The Canny edge detector involves a lot of stages involved in it. It uses a filter based on the derivative of a Gaussian in order to compute the intensity of the gradients. The Gaussian reduces the effect of noise present in the image. Then, potential edges are suppressed to 1-pixel curves by removing nonmaximum pixels of the gradient magnitude. Finally, edge pixels are kept or removed using hysteresis thresholding on the gradient magnitude. The Canny has three adjustable parameters: the width of the Gaussian (the noisier the image, the greater the width), and the low and high threshold for the hysteresis thresholding. (http://scikitimage.org/docs/dev/auto_examples/plot_canny.html) The first thing it does is to first smoothen the image to eliminate noise. It then finds the image gradient to highlight regions with high spatial derivatives. The algorithm then tracks along these regions and suppresses any pixel that is not at the maximum (non-maximum suppression). The gradient array is now further reduced by hysteresis. The remaining pixels that have not been suppressed are tracked by hysteresis. It makes use of two thresholds and if the magnitude is below the first threshold, it is set to zero (made a non-edge). However, if the magnitude is above the high threshold, it is made an edge but if the magnitude is between the 2 thresholds, it is set to zero unless there is a path from one pixel to another pixel with a gradient above the higher threshold.

From the above-listed edge detectors, it was observed that Canny yields better results because of the processes it adopts for the process of detecting edges. It takes very strong cognizance of the noise in the images and first works on the image to ensure acquisition of higher intensity pixels of the images. Even though it takes more time because of the processes involved, the trade-off between time and efficiency is a positive one. Considering the simplicity of using Sobel along with its ability to detect edges and orientations of the required images, it is relatively reliable and can be used where identification is all there is to the system being designed. However, for authentication and more efficiency in identifying an individual, Canny is a better method.

After the edge detection stage, features that are unique, repeatable and relatively stable are extracted. For this purpose, the Crossing Number (CN) is employed. This concept would be adopted with the use of the image where the palm-vein pattern is eight-connected.

The following are the major tasks that would form this stage:

a) minutiae extraction by scanning the local neighbourhood of each ridge pixel in the image using a $3 \times 3$ window.

b) computation of $\mathrm{CN}$ value; which is defined as half the sum of the differences between 
pairs of adjacent pixels in the eightneighbourhood.

identification of palm-vein features with 3 one-value neighbors as a vein bifurcation and those with 1 one-value neighbor as vein endings. (Rajalakshmi et al, 2011)

The $\mathrm{CN}$ for a ridge pixel $\mathrm{P}$ is given by:

$$
\sum_{\mathrm{CN}=0.5}^{8}\left|\boldsymbol{P}_{i}-\boldsymbol{P}_{i+1}\right|
$$

where $P_{i}$ is the pixel value in the neighbor-hood of $\mathrm{P}$. For a pixel $\mathrm{P}$, its eighth neighbouring pixels are scanned into an anti-clockwise direction.

\subsection{Feature Matching}

This stage requires much of digital signal processing because it is where the actual verification takes place. There are various methods for matching the previously enrolled palm-vein features and the palm-vein at the point of identification or authentication.

For example, the similarity of two palm-vein templates can be calculated by the number of features that match between the two images. Let $G$ be the enrolled palm-vein template and $\mathrm{L}$ be the palm-vein at the point of identification, the matching score of $\mathrm{G}$ and $\mathrm{L}$ can be calculated as

$$
F(G, L)=\frac{1}{C x D} \sum_{i=1}^{C} \sum_{j=1}^{D}[\overline{G(i, j) \oplus L(i, j)}]
$$

where $\mathrm{C} \times \mathrm{D}$ is the size of $\mathrm{G}$ or $\mathrm{L}, \oplus$ is the logical "exclusive or" operation. The issue of difference in the orientations of the palm-vein features is taken care of by translating the template $\mathrm{G}$ both vertically and horizontally by some points. Then the highest matching score of all translated positions is taken.

\section{Comparison/Analysis}

By using different edge detection methods for the same set of palm-vein features the following observations were made the false acceptance rate and false rejection rate metrics.

\section{Table 1. FAR and FRR table for Canny, Sobel} and Prewitt Edge Detection Methods

\begin{tabular}{|c|c|c|c|c|c|c|}
\hline $\begin{array}{c}\text { Palm- } \\
\text { print } \\
\text { ID }\end{array}$ & \multicolumn{2}{|c|}{ Canny } & \multicolumn{2}{c|}{ Sobel } & \multicolumn{2}{c|}{ Prewitt } \\
\cline { 2 - 7 } & $\begin{array}{c}\text { FAR } \\
(\%)\end{array}$ & $\begin{array}{c}\text { FRR } \\
(\%)\end{array}$ & $\begin{array}{c}\text { FAR } \\
(\%)\end{array}$ & $\begin{array}{c}\text { FRR } \\
(\%)\end{array}$ & $\begin{array}{c}\text { FAR } \\
(\%)\end{array}$ & $\begin{array}{c}\text { FRR } \\
(\%)\end{array}$ \\
\hline 1 & 0.02 & 1.2 & 0.1 & 3.9 & 0.03 & 2.2 \\
\hline 2 & 0.03 & 1.0 & 1.0 & 1.1 & 0.05 & 1.3 \\
\hline 3 & 0.1 & 1.5 & 0.3 & 1.0 & 1.2 & 2.2 \\
\hline 4 & 0.01 & 1.0 & 0.6 & 0.09 & 0.09 & 1.4 \\
\hline 5 & 0.1 & 2.02 & 0.12 & 4.0 & 2.5 & 4.02 \\
\hline
\end{tabular}

This further strengthens the decision to make use of the Canny Edge Detection Method at the feature extraction stage.

\section{Conclusion}

From the work above, it is clear that image processing involves many stages that employ different methods. These methods vary in the results depending on the angle from which the purpose of the processing is for. There are times that compromises need to be reached for the actual work to be done. Some trade-offs such as time and size of image; which eventually affects the size of the database. Hence, there is ample room for contributions from various sectors, depending on the preferred advantage.

\section{References}

[1] R.Derakhshani and A. Ross, “A Texture-Based Neural Network Classifier for Biometric Identification using Ocular Surface Vasculature," Appeared in Proc. Of International Joint Conference on Neural Networks (IJCNN), (Orlando, USA), 2007

[2] A. Shrotri, S.C. Rethrekar, M.H. Patil., B. Debnath and T. Kim, "Infrared Imaging of Hand Vein Patterns for Biometric Purposes," Journal of Security Engineering, 2009.

[3] X. Wua, D. Zhangb, K. Wanga and B. Huanga, "Palmprint classification using principal lines," School of Computer Science and Technology, Harbin Institute of Technology (HIT), Harbin 150001, China Department of Computing, Biometric Research Centre, Hong Kong Polytechnic University, Hung Hum, Kowloon, Hong Kong, Received 30 January 2004; accepted 12 February 2004.

[4] A. Nadort, "The Hand Vein Pattern Used as a Biometric Feature", A Master Literature Thesis,Free University, AmsterdamAmsterdam,May 2007.

[5] K. Wong, T. Lai, B. Lee and F. Shum, "Analysis of Palm Vein Biometric".

[6] International Telecommunication Union, "Biometrics and Standards", ITU-T Technology Watch Report. 2009.

[7] E. Sridevi., B. Aruna and P. Sowjanya, "An Exploration of Vascular Biometrics", Freshman Engineering Department, K.L. University, India.

[8] C Deepika, C. Lakshmi, A. Kandaswamy and C. Vimal, "Protection of Patient Identity and Privacy using Vascular Biometrics" International Journal of Security (IJS), Volume (4): Issue (5), 2009.

[9] L. Wang, G. Leedham and S.Y. Cho, "Infrared imaging of hand vein patterns for biometric purposes", IET Comput. Vis., Vol. 1, No. 3-4, 2007. 
[10] http://www.tiburon-ent.com), Tiburon Enterprises, Accessed on the 13th of June, 2013.

[11] O.A. Akinsowon, O.S. Adewale, B.K. Alese, “ Design of A Fingerprint Authentication System For Access Control", Tibiscus University of Timişoara, România, Anale. Seria Informatică. Vol. X fasc. 1 - 2012, Annals. Computer Science Series. 10th Tome 1st Fasc. - 2012, pp 78-84.

[12] R. Fisher, S. Perkins, A. Walker and E. Wolfart, "Feature Detectors - Sobel Edge", 2003.

[13] J.P Gomes Abel, "Visual Computing and Multimedia," Lab. 3 Edge Detectors, 2011, www.uweb.ucsb.edu/ shahnam/AfED.doc

[14] P.S. Rao, " Invariant Recognition of Broken Rectangular Biscuits with Fuzzy Moment Descriptors," Department of Electronics \& Telecommunications Engineering Jadavpur University, Kolkata-700032, 2010.

[15] B.S. Panigrahi, H.N. Pratihari, Dr. G.Devi G and D. Satyabrata, "A Palmprint Feature Extraction and Pattern Classification Based on Hybri PSO-K-Means Clustering," ISSN: 2229-4333 (Print) | ISSN: 0976-8491 (Online ), www.ijcst.com, IJCST Vol. 2, Issue 2, June 2011.

[16] V. Saini and R. Garg, "A Comparative Analysis on Edge Detection Techniques Used in Image Processing," IOSR Journal of Electronics and

Communication Engineering (IOSRJECE) ISSN: 22782834 Volume 1, Issue 2 (May-June 2012), PP 56-59, www.iosrjournals.org

[17] M.Juneja and S.S.Parvinder, "Performance Evaluation of Edge Detection Techniques for Images in Spatial Domain," International Journal of Computer Theory and Engineering, Vol. 1, No. 5, December, 2009, 17938201.

[18] Mohammed Namzul Haque, "Conference on Electronics and Telecommunication 2010 Bangladesh Electronic Society Implementation of a FPGA based Architecture of Prewitt Edge detection Algorithm using Verilog HDL," Department of Computer Science \& Engineering Daffodil International University, Dhaka, Bangladesh, e-mail: nazmul@daffodilvarsity.edu.bd.

[19]http://www.roborealm.com/help/Prewitt.php, Accessed on 31st July, 2013. 